DOI: $10.5216 /$ cab.v12i1.4098

\title{
UTILIZAÇÃO DE SISTEMAS DE REFRIGERAÇÃO DE SÊMEN EQUINO NA ESTABILIZAÇÃO DAS AMOSTRAS SEMINAIS PREVIAMENTE À CONGELAÇÃO
}

\author{
Bruna De Vita, ${ }^{1}$ Gabriel Barcellos Felício, ${ }^{1}$ Cely Marini Melo, ${ }^{1}$ José Antonio Dell’ Aqua Júnior ${ }^{1}$ e \\ Frederico Ozanam PaPA ${ }^{1}$
}

1. Departamento de Reprodução Animal e Radiologia Veterinária, Área de Reprodução animal - UNESP. E-mail: brudev@gmail.com

\begin{abstract}
RESUMO
Com este estudo objetivou-se analisar a eficiência da utilização de sistemas de transporte de sêmen refrigerado na estabilização das palhetas previamente à congelação de sêmen equino. Os ejaculados foram diluídos em meio diluente Botu-Sêmen ${ }^{\circledR}$, e centrifugados por dez minutos a $600 \mathrm{xg}$ e; os pellets foram ressuspendidos no diluente para criopreservação Botucrio ${ }^{\circledR}$, envasados em palhetas de $0,5 \mathrm{ml}$ e divididos em três grupos. No Grupo M, as palhetas foram dispostas horizontalmente em geladeira Minitub ${ }^{\circledR}$, a $5{ }^{\circ} \mathrm{C}$, por vinte minutos. Nos Grupos B e E, as palhetas foram colocadas respectivamente nos sistemas Botutainer ${ }^{\circledR}$ e Equitainer ${ }^{\circledR}$, previamente equilibrados na temperatura interna de $5^{\circ} \mathrm{C}$, e mantidas nesses sistemas também por vinte minutos. A congelação seguiu-se da mesma maneira para os três grupos. As palhetas foram descongeladas em banho-maria a $46^{\circ} \mathrm{C}$, por vinte segundos, e anali-

sadas quanto às motilidades total (MT) e progressiva (MP) (CASA) e integridade de membrana por microscopia de fluorescência (IMP). Não se observaram diferenças estatíscas $(\mathrm{P}>0,05)$ para os valores de $\mathrm{MT}\left(\mathrm{GM}=67,1 \%\right.$ a $; \mathrm{GB}=65,2 \%^{\mathrm{a}}$ e $\left.\mathrm{GE}=63,4 \% \mathrm{a}\right)$ e $\mathrm{MP}(\mathrm{GM}$ $=32,1 \%$ a $; \mathrm{GB}=31,9 \%^{\mathrm{a}}$ e $\left.\mathrm{GE}=30,4 \%^{\mathrm{a}}\right)$. Para os valores de IMP não foram observadas diferenças estatísticas entre os Grupos $\mathrm{M} \mathrm{e}$ $\mathrm{B}$; no entanto, o Grupo E apresentou um valor significativamente inferior $(\mathrm{P}<0,05)$ em comparação com os demais $(\mathrm{GM}=49,4 \%$, $\left.\mathrm{GB}=48,7 \%^{\mathrm{a}}, \mathrm{GE}=44,9 \%^{\mathrm{b}}\right)$. Apesar do sistema Equitainer ${ }^{\circledR}$ ser menos eficiente na manutenção da IMP do que os outros sistemas estudados, os dispositivos de transporte refrigerado de sêmen podem ser empregados na estabilização das amostras seminais previamente à congelação como uma alternativa aos refrigeradores, facilitando o procedimento de criopreservação a campo.
\end{abstract}

PALAVRAS-CHAVES: Equino, sêmen, estabilização, criopreservação.

\section{ABSTRACT}

\section{USE OF EQUINE SEMEN COOLING SYSTEMS TO STABILIZE SEMINAL SAMPLES BEFORE FREEZING}

The aim of this study was to evaluate the efficiency of passive cooling device to stabilize equine straws before freezing. The samples were diluted in Botu-Sêmen ${ }^{\circledR}$ extender and centrifuged at $600 \mathrm{xg}$ for 10 minutes. Then, the pellets were ressuspended with Botucrio $^{\circledR}$ cryopreservation extender, loaded into $0.5 \mathrm{~mL}$ straws and divided into three groups. On Group M, straws were placed horizontally in a Minitub ${ }^{\circledR}$ refrigerator at $5{ }^{\circ} \mathrm{C}$ for 20 minutes. For groups $\mathrm{B}$ and $\mathrm{E}$, the straws were placed in Botutainer ${ }^{\mathbb{B}}$ and Equitainer ${ }^{\circledR}$ devices, respectively. Both systems were previously stabilized at $5^{\circ} \mathrm{C}$ and straws were maintained for 20 minutes, and then frozen. The straws were thawed at $46{ }^{\circ} \mathrm{C}$ for 20 seconds. Sperm samples were evaluated by CASA for Total Motility (TM) and Progressive Motility (PM) and by fluorescent microscopy for Plasma Membrane Integrity (MPI). Statistical differences were not observed ( $p>0.05$ ) for Total Motility $(\mathrm{GM}=67.1 \%$; $\mathrm{GB}=65.2 \%$ and $\mathrm{GE}=63.4 \%)$ and Progressive Motility $(\mathrm{GM}=32.1 \%$; $\mathrm{GB}=31.9 \%$ and $\mathrm{GE}=30.4 \%)$. Although MPI percentages were not different between groups $\mathrm{M}$ and $\mathrm{B}$, group $\mathrm{E}$ showed a decrease in comparison to the other groups 
$(\mathrm{GM}=49.4 \%, \mathrm{~GB}=48.7 \%, \mathrm{GE}=44.9 \%)$. These results demonstrate that passive cooling devices can be used to stabilize equine straws

KEYWORDS: Equine, semen, stabilization, cryopreservation.

\section{INTRODUÇÃO}

O desenvolvimento de biotecnologias cada vez mais modernas tem contribuído para os avanços na área de Reprodução animal. A criopreservação de sêmen se destaca por suas diversas vantagens, como diminuição de custos com aquisição de animais geneticamente superiores; armazenamento por tempo indeterminado; utilização do sêmen de animais excepcionais mesmo após a perda da capacidade reprodutiva ou morte; racionalização do uso do reprodutor com aumento da relação macho-fêmea; controle de doenças venéreas e facilidade no transporte a longas distâncias. No entanto, a criopreservação encontra desafios como a variabilidade da congelabilidade de sêmen entre garanhões e o transporte de garanhões a centros especializados para congelação.

Alguns protocolos incluem um período de resfriamento do sêmen antes da congelação, com o objetivo de recuperar as células espermáticas dos efeitos da centrifugação e diminuir as lesões celulares que podem ter sido causadas pelo choque frio (JASKO, 1994). Pode-se evitar ou amenizar ocorrência do choque pelo frio refrigerando-se lentamente o sêmen equino diluído até $4{ }^{\circ} \mathrm{C}$, com taxas iguais ou inferiores a $0,05^{\circ} \mathrm{C} / \mathrm{min}$ (PICKETT \& AMANN, 1993).

O termo choque frio define algumas modificações que ocorrem no espermatozoide quando submetido a uma curva de refrigeração rápida (JASKO, 1994). Esse processo se caracteriza por uma alteração na orientação dos lipídeos de membrana induzindo um desarranjo das moléculas, com formação de pontos de fragilidade que levam a uma permeabilidade excessiva ou mesmo à ruptura da membrana, resultando em padrão anormal de movimento espermático, perda rápida de motilidade, danos no acrossoma, redução do metabolismo e perda dos componentes intracelulares (AMANN \& GRAHAM, 1992; KAYSER et al., 1992; SQUIRES et al., 1999).

A despeito da conhecida sensibilidade do espermatozoide equino ao choque pelo frio, alguns estudos foram desenvolvidos com o intuito de avaliar before freezing, being an alternative for replacing refrigerators, making easier the cryopreservation in the field.

a possibilidade de se congelar sêmen de garanhões sem estabilização refrigerada prévia. Em dois estudos - um realizado por VIDAMENT et al., (2000), em que se utilizou o diluente INRA 82, e outro, em que o sêmen foi congelado por FURST et al. (2003) em meio a uma base de lactose-EDTA-gema de ovo - houve queda significativa na motilidade pós-descongelação, quando não se realizou antes o período de estabilização refrigerada.

Com o intuito de permitir a colheita, diluição e pré-resfriamento do sêmen em transportes adequados para envio a laboratórios especializados na congelação, fizeram-se alguns estudos sobre a congelação de sêmen após um período de até 24 horas de refrigeração a 5 ${ }^{\circ} \mathrm{C}$ e $15{ }^{\circ} \mathrm{C}$ (CROCKET et al., 2001; BACKMANN et al., 2004; MELO et al., 2006). MELO et al (2006) observaram que a congelação de sêmen equino após 24 horas de refrigeração no sistema Equitainer ${ }^{\circledR}$ não interferiu nos parâmetros espermáticos de motilidade total e progressiva, bem como na integridade de membrana espermática e índices de fertilidade. Resultados semelhantes haviam sido observados anteriormente por BACKMANN et al. (2004), quando o sêmen foi congelado após dezoito horas de refrigeração. Porém esses resultados diferiram dos encontrados por CROCKET et al. (2001), os quais observaram uma redução na motilidade progressiva do sêmen refrigerado por 24 horas a $5{ }^{\circ} \mathrm{C}$ antes da congelação.

Esses sistemas passivos são muito utilizados no Brasil e no mundo no emprego do sêmen refrigerado, e muitos estudos demonstraram sua eficiência na manutenção da viabilidade seminal (BATELLIER et al., 1997; MACHADO et al., 2002; BRINSKO et al., 2003; FARRÁS et al., 2008; NUNES et al. 2008). A biotecnologia permite ao proprietário do garanhão maior flexibilidade para colher e enviar o sêmen no momento oportuno, tendo sido demonstrados diversos avanços em sua utilização (PICKETT, 1992; AURICH, 2008).

No presente estudo, objetivou-se analisar a eficiência da utilização dos sistemas de transporte refrigerado de sêmen equino Equitainer ${ }^{\circledR}$ (Hamilton-Thorne Research Beverly/MA, USA) e Botutainer ${ }^{\circledR}$ (Biotech 
Botucatu, SP, Brasil) para a estabilização seminal previamente à congelação, em comparação com o uso do refrigerador programável Minitub ${ }^{\circledR}$ (Minitub do Brasil, Porto Alegre, Brasil) na manutenção da qualidade dos parâmetros espermáticos de MT, MP e IMP.

\section{MATERIAL E MÉTODOS}

Animais

Foram utilizados quatro garanhões com idades entre cinco e vinte anos, das raças Westfallen, Quarto de Milha, Puro Sangue Árabe e Hannoverano, pertencentes ao Centro de Reprodução e Biotecnologia Equina (Cerbeq) e Departamento de Reprodução animal e Radiologia Veterinária/FMVZ/Unesp, Botucatu. Utilizaram-se nove ejaculados dos garanhões das raças Westfallen e Hannoverano, e nove ejaculados dos garanhões das raças Quarto de Milha (QM) e Puro Sangue Árabe (PSA) totalizando 36 ejaculados. Porém, seis amostras (três QM, e três PSA) foram desprezadas pela dificuldade de armazenamento das palhetas no sistema Equitainer ${ }^{\circledR}$.

Colheita de sêmen

A colheita de sêmen foi realizada com o uso de vagina artificial (Biotech Botucatu, SP, Brasil). Esta era composta por um tubo rígido de fibra de vidro, mucosa de látex e mucosa plástica descartável, acoplada a um copo coletor isotérmico contendo uma camisa sanitária descartável, na temperatura de aproximadamente $45^{\circ} \mathrm{C}$.

\section{Análises laboratoriais}

Filtrou-se cada ejaculado com auxílio de um filtro de nylon e mensurou-se o volume em proveta graduada. Em seguida, foi feita a avaliação macroscópica quanto à cor, ao odor e ao aspecto, com o objetivo de eliminar amostras que apresentassem alguma anormalidade. Uma pequena alíquota foi retirada para avaliação computadorizada (CASA) dos parâmetros de motilidade total e progressiva (Hamilton-Thorne Research Beverly/MA, USA) e concentração. Essa análise foi realizada em microscópio de luz com contraste de fase (200x), com auxílio de uma câmara de
Neubauer após diluição na proporção de 1:20 (sêmen: água destilada).

Processamento do sêmen

Diluíram-se os ejaculados na proporção de 1:1 com diluente Botu-Sêmen ${ }^{\circledR}$ (Biotech Botucatu, SP, Brasil) e centrifugaram-se por dez minutos a $600 \mathrm{xg}$. Os pellets foram ressuspendidos com diluente para criopreservação Botu-Crio ${ }^{\circledR}$ (Biotech Botucatu, SP, Brasil), na concentração de $200 \times 10^{6}$ espermatozoides viáveis por $\mathrm{mL}$, envasados em palhetas de $0,5 \mathrm{~mL}$ e divididos em três grupos. No Grupo M (GM), as palhetas foram dispostas horizontalmente em geladeira Minitub ${ }^{\circledR}(\mathrm{Mi}-$ nitub do Brasil, Porto Alegre, Brasil) a $5{ }^{\circ} \mathrm{C}$ por vinte minutos. No Grupo B (GB), colocaram-se as palhetas verticalmente no sistema de transporte refrigerado de sêmen Botutainer ${ }^{\circledR}$ (Biotech Botucatu, SP, Brasil), previamente equilibrado na temperatura interna de 5 ${ }^{\circ} \mathrm{C}$, onde foram mantidas por vinte minutos. No Grupo $\mathrm{E}$ (GE), as palhetas foram dispostas verticalmente no sistema Equitainer ${ }^{\circledR}$ (Hamilton Research INC., USA), também previamente equilibrado na temperatura interna de $5{ }^{\circ} \mathrm{C}$, e mantidas pelos mesmos vinte minutos.

A maior dificuldade encontrada na utilização do sistema Equitainer ${ }^{\circledR}$, neste experimento, foi o armazenamento das palhetas em seu interior, já que a altura do recipiente interno desse sistema é menor do que a altura das palhetas de $0,5 \mathrm{~mL}$ utilizadas, ocorrendo o dobramento de algumas amostras, que tiveram que ser desprezadas. No sistema Botutainer ${ }^{\circledR}$, não houve dificuldade no armazenamento das palhetas, visto que ele foi desenvolvido e adaptado para palhetas de 0,5 e $0,25 \mathrm{~mL}$.

Após o período de estabilização, a congelação seguiu-se da mesma maneira para os três grupos. As palhetas foram colocadas horizontalmente a $6 \mathrm{~cm}$ acima do nível de nitrogênio líquido $\left(\mathrm{N}_{2}\right)$ em caixa de isopor de 40 litros, com dimensões de $40 \times 30$ x $25 \mathrm{~cm}$, contendo $3,5 \mathrm{~cm}$ de $\mathrm{N}_{2}$, foram mantidas em vapor de $\mathrm{N}_{2}$ por vinte minutos, e depois foram criopreservadas por imersão direta (PAPA et al., 2008).

Descongelaram-se as palhetas em banho-maria a $46^{\circ} \mathrm{C}$, por vinte segundos (DELL'AQUA JR. 2000), e então foi realizada a análise computadorizada do sêmen (CASA) quanto às motilidades total e progressiva (Hamilton-Thorne Research Beverly/MA, USA) e 
integridade de membrana plasmática por microscopia de fluorescência, através da associação das sondas iodeto de propídeo e diacetato de 6-carboxifluoresceína (HARISSON \& VICKERS, 1990).

Para a comparação dos efeitos do sistema de refrigeração na estabilização de amostras previamente à congelação, foi realizada a análise de variância (ANOVA). Nos casos em que foi verificada variação, utilizou-se o teste de Tukey para avaliar as diferenças significativas entre os grupos.

\section{RESULTADOS E DISCUSSÃO}

A média e o desvio padrão dos parâmetros de motilidade total (MT) e motilidade progressiva (MP) obtidos pela avaliação computadorizada (CASA) das amostras de sêmen equino a fresco antes do procedimento de estabilização e congelação foram de $86,5 \pm$ $5,5 \%$ e $38,3 \pm 9$, respectivamente.

No presente estudo não houve diferença significativa entre os valores dos parâmetros espermáticos de motilidade total e motilidade progressiva das amostras quando comparadas as diferentes formas de estabilização. No entanto, quando avaliada a integridade de membrana plasmática, o sistema Equitainer ${ }^{\circledR}$ apresentou-se menos eficiente em relação aos outros sistemas estudados $(\mathrm{p}<0,05)$. MELO et al. (2006) utilizaram o sistema Minitub $^{\circledR}$ para o período de estabilização das amostras seminais durante vinte minutos a $5{ }^{\circ} \mathrm{C}$ e obtiveram resultados de motilidades total e progressiva (68,9\% e 31,6\%) similares aos do presente experimento (GM: 67,1\% e 32,1\%; GB: 65,2\% e 31,9\%; GE: 63,4\% e $30,4 \%$ - Tabela 1$)$.

TABELA 1. Médias e desvios padrão (\%) dos parâmetros espermáticos de motilidade total (MT), motilidade progressiva (MP) e integridade de membrana plasmática (IMP) das amostras de sêmen equino a fresco e descongeladas, submetidas ao período de estabilização prévio à congelação nos sistemas Minitub ${ }^{\circledR}$, Botutainer $^{\mathbb{R}}$ e Equitainer ${ }^{\mathbb{R}}$

\begin{tabular}{lccc}
\hline \multicolumn{4}{c}{ Sistemas } \\
\hline Variáveis & Minitub $^{\circledR}$ & Botutainer $^{\circledR}$ & Equitainer $^{\circledR}$ \\
\hline MT & $67,1 \pm 10,34$ & $65,2 \pm 10,63$ & $63,4 \pm 11,45$ \\
MP & $32,1 \pm 7,84$ & $31,9 \pm 6,71$ & $30,4 \pm 7,27$ \\
IMP & $49,4 \pm 5,43^{\text {a }}$ & $48,7 \pm 7,12^{\text {a }}$ & $44,9 \pm 6,74^{\mathrm{b}}$ \\
\hline
\end{tabular}

Médias seguidas de distintas, letras na mesma linha, diferem estatisticamente $(\mathrm{p}<0,05)$.
Ainda em relação à integridade da membrana plasmática, seria esperado que não houvesse diferença entre os grupos testados, especialmente entre os sistemas Botutainer ${ }^{\circledR}$ e Equitainer ${ }^{\circledR}$, uma vez que as curvas de refrigeração são bastante semelhantes entre eles. Ao se compararem os sistemas Botutainer ${ }^{\circledR} \mathrm{e}$ Equitainer ${ }^{\circledR}$ sob diferentes temperaturas, simulando-se estresse térmico pelo frio $\left(-4^{\circ} \mathrm{C}\right)$, pelo calor $\left(38^{\circ} \mathrm{C}\right) \mathrm{e}$ pela temperatura ambiente $\left(24^{\circ} \mathrm{C}\right)$, diferentemente do que se observou no presente experimento, não foram encontradas diferenças significativas da integridade de membrana plasmática entre os sistemas testados (AVANZI et al., 2006; AVANZI et al., 2007).

Apesar da manutenção da viabilidade espermática, as taxas de refrigeração utilizadas no presente estudo $\left(3,4^{\circ} \mathrm{C} / \mathrm{min}\right.$ no refrigerador programável e $1,13^{\circ} \mathrm{C} /$ min nos sistemas de transporte) foram diferentes das taxas recomendadas por KAYSER et al (1992). Esses autores concluíram que o espermatozoide equino pode ser refrigerado rapidamente de 37 para $20^{\circ} \mathrm{C}$, mas deve ser refrigerado a uma taxa menor que $0,1^{\circ} \mathrm{C}$ por minuto, preferencialmente a $0,05^{\circ} \mathrm{C}$ por minuto, de 20 para $5{ }^{\circ} \mathrm{C}$, para manter a máxima sobrevivência espermática. Os sistemas de transporte refrigerado Equitainer ${ }^{\circledR} \mathrm{e}$ Botutainer ${ }^{\circledR}$ apresentam taxas de refrigeração de 0,03 e $0,05{ }^{\circ} \mathrm{C} / \mathrm{min}$, respectivamente, e são considerados adequados à biotecnologia de sêmen refrigerado. Provavelmente essas taxas não foram atingidas devido ao volume interno após a estabilização ser inferior ao indicado pelos fabricantes. Acontece que o presente estudo foi delineado com base em resultados obtidos para a espécie bovina, cujas palhetas são envasadas na fazenda, transportadas no sistema Botutainer ${ }^{\circledR}$ por até 48 horas e congeladas no laboratório, sem prejuízos nos índices de fertilidade (CRESPILHO, 2010).

Apesar de as curvas utilizadas no presente trabalho serem mais rápidas do que as recomendadas por KAYSER et al. (1992), as três metodologias testadas permitiram a obtenção de bons resultados de motilidade total $(\mathrm{GM}=67,1 \%, \mathrm{~GB}=65,2 \%, \mathrm{GE}=63,4 \%)$, motilidade progressiva $(\mathrm{GM}=32,1 \%, \mathrm{~GB}=31,9 \%$, $\mathrm{GE}=30,4 \%$ ) e integridade de membrana plasmática $\left(\mathrm{GM}=49,4 \%^{\mathrm{a}}, \mathrm{GB}=48,7 \%^{\mathrm{a}}, \mathrm{GE}=44,9 \%{ }^{\mathrm{b}}\right), \mathrm{de}-$ monstrando que os três dispositivos foram eficientes na estabilização seminal prévia à congelação. Essa similaridade talvez possa ser explicada pelo fato de os animais utilizados apresentarem sêmen de alta con- 
gelabilidade e pelo uso do meio diluente Botu-Crio ${ }^{\circledR}$. Este tem demonstrado oferecer maior proteção às células espermáticas durante o processo de congelação de sêmen equino em comparação aos diluentes mais utilizados no mundo - INRA 82 e Lactose-EDTA-gema de ovo, como descrito por PASQUINI et al. (2007), e às amostras de sêmen de epidídimo de garanhões, conforme PAPA et al. (2008). Ademais, a presença de substâncias com a função de crioproteção, seja intra ou extracelular, como açúcares, gema de ovo, glicerol e metilformamida, contidas no diluente Botu-Crio ${ }^{\circledR}$, conferem ao espermatozoide maior proteção contra uma curva de refrigeração mais rápida.

\section{CONCLUSÃO}

Com base nos resultados obtidos e nas condições em que se realizou o presente trabalho, foi possível concluir que os sistemas Botutainer $^{\circledR}$ (GB) e Minitub ${ }^{\circledR}$ (GM) foram mais eficientes quanto à manutenção da integridade de membrana plasmática em relação ao Equitainer $^{\circledR}(\mathrm{GE})$. No entanto, os sistemas de transporte refrigerado de sêmen equino Botutainer ${ }^{\circledR}$ e Equitainer ${ }^{\circledR}$ podem ser utilizados para a realização do período de estabilização previamente à congelação, pois foram obtidos valores de MT, MP e IMP pós-descongelação tão satisfatórios quanto os obtidos utilizando-se o refrigerador programável Minitub ${ }^{\circledR}$, facilitando assim a etapa da estabilização $\left(5^{\circ} \mathrm{C} / 20 \mathrm{~min}\right)$ e, consequentemente, a congelação de sêmen equino a campo.

\section{AGRADECIMENTO}

\section{Ao CNPq.}

\section{REFERÊNCIAS}

AMANN, D. P.; GRAHAM, J. K. Spermatozoal function. In: McKINNON, A. O.; VOSS, J. L. (Eds.). Equine reproduction. Philadelphia: Lea \& Febiger, 1992. p. 715-745.

AURICH, C. Recent advances in cooled-semen technology. Animal Reproduction Science, v. 107, p. 268-275, 2008.

AVANZI, B. R.; PAPA, F. O.; RAMOS, S. A.; FARRAS, M. C.; MELO, C. M.; ALVARENGA, M. A.; DELL'AQUA JR., J. A; MEDEIROS, A. S. L. Efficiency of different cooling and storage systems for maintaining equine semen viability in a hot environment. Animal Reproduction Science, v. 94, p. 152-154, 2006.
AVANZI, B. R.; PAPA, F. O.; RAMOS, S. A.; FARRAS, M. C.; MELO, C. M.; ALVARENGA, M. A.; DELL'AQUA JR., J. A; MEDEIROS, A. S. L. Avaliação de diferentes sistemas de transporte de sêmen equino refrigerado na manutenção da viabilidade espermática. Acta Scientiae Veterinariae, v. 35, (supl. 3), p. 1.310, 2007.

BACKMAN, T.; BRUEMMER, J. E.; GRAHAM, J. K.; SQUIRES, E. L. Pregnancy rates of mares inseminated with semen cooled for 18 hours and then frozen. Journal of Animal Science, v. 82, p. 690-694, 2004.

BATELLIER, F.; MAGISTRINI, M.; FAUQUANT, J.; PALMER, E. Effect of milk fractions on survival of equine spermatozoa. Theriogenology, v. 48, p. 391-410, 1997.

BRINSKO, S. P.; BLANCHARD, T. L.; RIGBY, S. L.; LOVE, C. C; VARNER, D. D. Effects of dead spermatozoa on motion characteristics and membrane integrity of live spermatozoa in fresh and cooled-stored equine semen. Theriogenology, v. 59, p. 735-742, 2003.

CRESPILHO, A. M. Estudo comparativo de diferentes metodologias de preservação do sêmen bovino para a utilização em programas de inseminação artificial em tempo-fixo (IATF). 2010. 124 f. Tese (Doutorado) - Faculdade de Medicina Veterinária e Zootecnia, Universidade Estadual Paulista, Botucatu, 2010. Disponível em: $<$ http://www.athena.biblioteca.unesp.br $>$. Acesso em: ?????

CROCKET, E. C.; GRAHAM, J. K.; BRUEMMER, J. E.; SQUIRES, E. L. Effect of cooling equine spermatozoa before freezing on post-thawmotility: preliminary results. Theriogenology, v. 57, p. $793-803,2001$.

DELL'AQUA JR., J. A. Efeito da centrifugação, tipos de envase e temperatura de descongelação sobre os parâmetros espermáticos e índices de fertilidade relacionados com o local de deposição e concentração da dose inseminante do sêmen congelado equino. 2000. 81 f. Dissertação (Mestrado) - Faculdade de Medicina Veterinária e Zootecnia, Universidade Estadual Paulista, Botucatu, 2000. Disponível em: <http://www.athena.biblioteca. unesp.br>. Acesso em:?????

FARRÁS, M. C.; AVANZI, B. R.; MELO, C. M.; DELL'AQUA JR., J. A.; PAPA, F. O. Efeito de diferentes diluentes na manutenção das características do sêmen equino em dois sistemas de refrigeração passiva. Ciência Animal Brasileira, v. 9, n. 3, p. 693-699, 2008.

FURST, R.; CARVALHO, G. R.; FURST, M. C. O.; MAFILLI, V. V.; PROSPERI, C. P.; LIMA NETO, A. Efeito do resfriamento na criopreservação do sêmen equino. Revista Brasileira de Reprodução animal, v. 27, n. 3, p. 348, 2003. 
HARRISON, R. A.; VICKERS, S. E. Use of fluorescent probes to assess membrane integrity in mammalian spermatozoa. Journal of Reproduction and Fertility, v. 88, p. 343-352, 1990.

JASKO, D. J. Procedures for cooling and freezing of equine semen. Ars Veterinaria, v. 10, n. 2, p. 156-165, 1994.

KAYSER, J. P.; AMANN, R. P.; SHIDELER, E. L.; JASKO, D. L.; PICKETT, B. W. Effect of linear cooling rate on motion characteristics of stallion spermatozoa. Theriogenology, v. 38, p. 601-614, 1992.

MACHADO, M. S.; LEÃO, K. M.; GOMES, G. M.; MACEDO, L. P.; ALVARENGA, M. A. Efeitos de diferentes sistemas de transporte sobre a qualidade do sêmen refrigerado equino. Revista Brasileira de Reprodução animal, v. 26, p. 194-196, 2002.

MELO, C. M.; ALVARENGA, M. A.; ZANH, F. S.; MARTIN, I.; ORLANDI, C.; TRINQUE, C. L. A.; DELL'AQUA JR., J. A.; PAPA, F. O. Effect of the transport container and cryoprotectant on freezability of equine semen previously cooled for $24 \mathrm{~h}$. Animal Reproduction Science, v. 94, p. 78-81, 2006.

NUNES, D. B.; ZORZATTO, J. R.; COSTA E SILVA, E. V.; ZÚCCARI, C. E. S. N. Efficiency of short-term storage of equine semen in a simple-design cooling system. Animal Reproduction Science, v. 104, p. 434-439, 2008.

PAPA, F. O.; MELO, C. M.; FIORATTI, E. G.; DELL'AQUA JR., J. A.; ZAHN, F. S.; ALVARENGA, M. A. Freezing of stallion epididymal sperm. Animal Reproduction Science, v. 107, p. 101-111, 2008.

PASQUINI, D. F.; FERREIRA, H.; PAPA, F. O.; DELL'AQUA JR., J. A.; MEDEIROS, A. S. L.; ALVARENGA, M. A. Resultados preliminares dos parâmetros espermáticos de sêmen equino congelado com três dos diluentes mais utilizados no mundo. Acta Scientiae Veterinariae, v. 35, (supl. 3), p. 1.007, 2007.

PICKETT, B. W. Seminal extenders and cooled semen. In: McKINNON, A. O; VOSS, J. L. (Eds.). Equine reproduction. Philadelphia: Lea \& Febiger, 1992. p. 746-789.

PICKETT, B. W.; AMANN, R. P. Cryopreservation of semen. In: McKINNOM, A. D.; VOSS, J. L. Equine reproduction. Malvern: Lea \& Febiger, 1993. p. 769-789.

VIDAMENT, M.; ECOT, P.; NOUE, P.; BOURGEOIS, C.; MAGISTRINI, M.; PALMER, E. Centrifugation and addtion of glycerol at $22{ }^{\circ} \mathrm{C}$ instead of $4{ }^{\circ} \mathrm{C}$ improve post-thaw motility and fertility of stallion spermatozoa. Theriogenology, v. 54, p. 907-919, 2000.

Protocolado em: 23 jun. 2008. Aceito em: $1^{\text {o dez. }} 2010$. 